\title{
Investigation of the Electrophysiological Remodelling in Acute and Chronic Post- Myocardial Infarction
}

\author{
Xin Zhou ${ }^{1}$, Jakub Tomek ${ }^{2}$, Blanca Rodriguez ${ }^{1}$ \\ ${ }^{1}$ Department of Computer Science, University of Oxford, Oxford, UK \\ ${ }^{2}$ Department of Physiology, Anatomy and Genetics, University of Oxford, Oxford, UK
}

\begin{abstract}
Sudden cardiac death was reported in both the acute stage and the chronic heart failure (HF) stage after myocardial infarction (MI). The goal of this study is to investigate the pro-arrhythmic cellular effects of post-MI electrophysiological remodelling for both the acute stage and the chronic stage, using human-based computer modelling and simulation.

The ToR-ORd human ventricular cellular model was used as baseline, and a new formulation of the calcium activated potassium current was also included in order to reflect its enhanced expression in the HF stage. We constructed and calibrated populations of human ventricular cell models to represent electrophysiological variability of normal zone (NZ) myocytes. For the acute post-MI stage, three types of border zone (BZ) ionic remodelling were considered based on canine experimental data. For the chronic phase with the development of $H F$, ionic remodelling in $B Z$ and the remote zone (RZ) were introduced based on minipig and human experiments.

For both the acute stage and the chronic stage post-MI, simulations showed that both large and small proarrhythmic dispersion in action potential duration can be present between $B Z$ and non-infarcted $N Z$ or $R Z$, and the post-MI ionic remodelling led to weak calcium transient in the $B Z$.
\end{abstract}

\section{Introduction}

The incidence of sudden cardiac death (SCD) was reported to be highest within the first month after a myocardial infarction (MI), followed by a progressive decline to a plateau after a few months. Ventricular tachyarrhythmia accounts for about $50 \%$ of these SCD [1]. The electrophysiological heterogeneity in post-MI human cardiac tissue, such as the dispersion of action potential duration (APD) between normal zone (NZ) and border zone (BZ), provides substrates for the generation and maintenance of lethal reentrant waves. Therefore, it is crucial to understand the effects of post-MI ionic remodelling for both the acute and the chronic stages.

For the acute stage post-MI, experimental data from 4 to 5 days post-MI dogs showed reduced sodium current $\left(\mathrm{I}_{\mathrm{Na}}\right)[2]$, L-type calcium current $\left(\mathrm{I}_{\mathrm{CaL}}\right)[3]$, potassium currents $\left(\mathrm{I}_{\mathrm{Kr}}\right.$ and $\left.\mathrm{I}_{\mathrm{Ks}}\right)[4]$, and enhanced CaMKII phosphorylation [5] in the BZ. However, these aspects of ionic remodelling may recover in the healing process [6], and it is essential to consider different healing stages in the proarrhythmic risk investigations.

For the healed stage, one recent study conducted in minipigs investigated the electrophysiological remodelling of individual currents after 5 months post-MI with the progression towards heart failure (HF)[7]. In the $\mathrm{HF}$ remote zone (RZ) myocytes, decreased $\mathrm{I}_{\mathrm{Kr}}$, increased late sodium current $\left(\mathrm{I}_{\mathrm{NaL}}\right)$, calcium activated potassium and chloride currents $\left(\mathrm{I}_{\mathrm{KCa}}\right.$ and $\left.\mathrm{I}_{\mathrm{ClCa}}\right)$ were observed [7]. Similar remodelling also occurred in the BZ myocytes, and with additional decrease of $\mathrm{I}_{\mathrm{CaL}}$ and inward rectifier potassium current $\left(\mathrm{I}_{\mathrm{K} 1}\right)$.

Due to the lack of ionic remodelling data from human post-MI myocytes, these experimental data obtained from infarcted animals provide valuable insights to mimic human electrophysiological changes. The goal of this study is to explore the proarrhythmic effects of post-MI ionic remodelling in human ventricular myocyte models of electrophysiology based on experimental observations from both the acute and the chronic stages.

\section{Methods}

\subsection{The baseline model}

The ToR-ORd human ventricular cellular model [8] was used as the baseline model in this study. In order to introduce the enhanced activity of $\mathrm{I}_{\mathrm{KCa}}$ in $\mathrm{HF}$, a new formulation of the $\mathrm{I}_{\mathrm{KCa}}$ was added into the ToR-ORd model based on the data by [9], and the ratio of the current in the subspace (ss) was set to be the same as $\mathrm{I}_{\mathrm{CaL}}$. The conductance of the background potassium current was 
rescaled to $90 \%$ to adapt to the implementation of $\mathrm{I}_{\mathrm{KCa}}$. The formulation of the $\mathrm{I}_{\mathrm{KCa}}$ is the following:

gkca $=0.003$; ikcan $=3.5 ; \mathrm{kdikca}=6.05 \mathrm{e}-04$;

Fraction $\mathrm{I}_{\mathrm{KCa}} \mathrm{SS}=0.8$; FractionI $\mathrm{I}_{\mathrm{KCa}} \mathrm{i}=1-$ FractionI $_{\mathrm{KCaSS}}$;

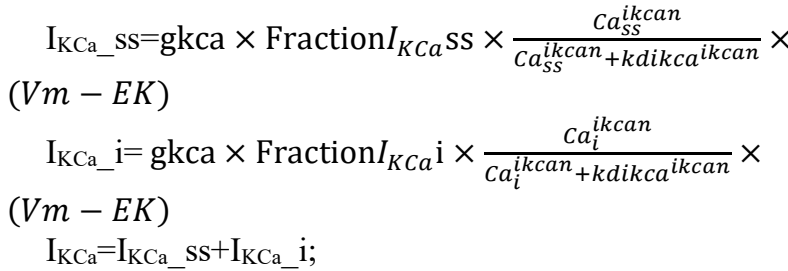

\subsection{Cell model population}

In order to simulate the effect of electrophysiological variability in human cardiomyocytes, an initial population of 500 human endocardial ventricular cell models was constructed based on the baseline model by varying $11 \mathrm{key}$ ionic currents by up to $\pm 50 \%$ as in previous studies [10]. After pacing at $1 \mathrm{~Hz}$ by 500 beats, the models were calibrated with human experimental data [11], [12]. 253 models were accepted and used as NZ population of models (POMs).

\subsection{Three types of $\mathrm{BZ}$ remodelling for the acute post-MI stage}

Three types of BZ remodelling for the acute post-MI stage were applied to the NZ based on previous publications [13]-[16].

Table 1. BZ ionic remodelling of individual currents in the acute stage post-MI.

\begin{tabular}{llll}
\hline Scaling factors & Acute BZ1 & Acute BZ2 & Acute BZ3 \\
\hline $\mathrm{I}_{\mathrm{Na}}$ & 0.4 & 0.38 & 0.4 \\
$\mathrm{I}_{\mathrm{to}}$ & 0.1 & & 0 \\
$\mathrm{I}_{\mathrm{CaL}}$ & 0.64 & 0.31 & 0.64 \\
$\mathrm{I}_{\mathrm{Kr}}$ & 0.7 & 0.3 & \\
$\mathrm{I}_{\mathrm{Ks}}$ & 0.2 & 0.2 & \\
$\mathrm{I}_{\mathrm{K} 1}$ & 0.3 & & 0.6 \\
$\mathrm{I}_{\mathrm{Cab}}$ & & & 1.33 \\
$\mathrm{aCaMK}$ & & & 1.5 \\
Tau_relp & & & $6 \neq$ \\
\hline
\end{tabular}

* Slower calcium release was introduced to reproduce effects of CaMKII activation in experiments.

\section{4. $\quad B Z$ and $R Z$ remodelling for the chronic post-MI HF stage}

The chronic post-MI BZ and one type of RZ remodelling were introduced based on [7] [17] [18]. Another type of RZ remodelling was established based on multiple experimental references[9], [17], [19]-[22].
Table 2. BZ and RZ ionic remodelling of individual currents in the chronic HF stage post-MI

\begin{tabular}{llll}
\hline $\begin{array}{l}\text { Scaling } \\
\text { factors }\end{array}$ & $\begin{array}{l}\text { Chronic } \\
\mathrm{BZ}\end{array}$ & $\begin{array}{l}\text { Chronic } \\
\mathrm{RZ1}\end{array}$ & $\begin{array}{l}\text { Chronic } \\
\mathrm{RZ2}\end{array}$ \\
\hline $\mathrm{I}_{\mathrm{Na}}$ & 0.43 & 0.43 & 0.43 \\
$\mathrm{I}_{\mathrm{NaL}}$ & 1.275 & 1.413 & 2 \\
$\mathrm{I}_{\mathrm{to}}$ & & & 0.6 \\
$\mathrm{I}_{\mathrm{CaL}}$ & 0.7 & & \\
$\mathrm{I}_{\mathrm{Kr}}$ & 0.89 & 0.87 & 0.6 \\
$\mathrm{I}_{\mathrm{Ks}}$ & & & 0.4 \\
$\mathrm{I}_{\mathrm{K} 1}$ & 0.76 & & 0.6 \\
$\mathrm{I}_{\mathrm{NaK}}$ & & & 0.6 \\
$\mathrm{~J}_{\mathrm{up}}$ & 0.4 & 0.4 & 0.3 \\
$\mathrm{I}_{\mathrm{KCa}}$ & 2 & 2 & $3.75^{*}$ \\
$\mathrm{I}_{\mathrm{ClCa}}$ & 1.25 & 1.25 & 1.25 \\
$\mathrm{aCaMK}$ & 1.5 & 1.5 & 1.5 \\
Tau_relp & 6 & 6 & 6 \\
\hline
\end{tabular}

* kdikca=3.45e-04 for Chronic RZ2

\section{Results}

\subsection{Variable APD dispersions in both the acute and the chronic post-MI stages}

For the acute stage, the three types of BZ remodelling led to different extent of APD prolongation in the baseline model (Figure 1, left). The Acute BZ1 and Acute BZ2 remodelling caused significant APD prolongation in both the endocardial and epicardial BZs. The Acute BZ2 remodelling also resulted in a significant reduction in the action potential (AP) plateau level. On the other hand, the Acute BZ3 remodelling caused the mildest change in both endocardial and epicardial models. In the population of 253 models, the Acute BZ2 remodelling group tended to show the longest APDs, while the Acute BZ3 remodelling group displayed similar APDs with the NZ group in both the epicardial and endocardial POMs (Figure 1, right).

For the chronic post-MI stage with HF, BZ and two types of RZs all led to longer APD than NZ, and the prolongation was more significant in the endocardial model (Figure 2, left). Chronic BZ and Chronic RZ1 had similar extent of APD prolongation, especially in the epicardium, corresponding to very small APD dispersions. However, the APD dispersion between the Chronic RZ2 and the Chronic BZ was much bigger due to a more significant APD prolongation in this type of RZ (Figure 2, left). The population of models showed similar trend as the baseline model, with APD prolongation most significant in the Chronic RZ2 (Figure 2, right).

Therefore, for both the acute stage and the chronic stage post-MI, variable APD dispersions can exist depending on the types of ionic remodelling present. 

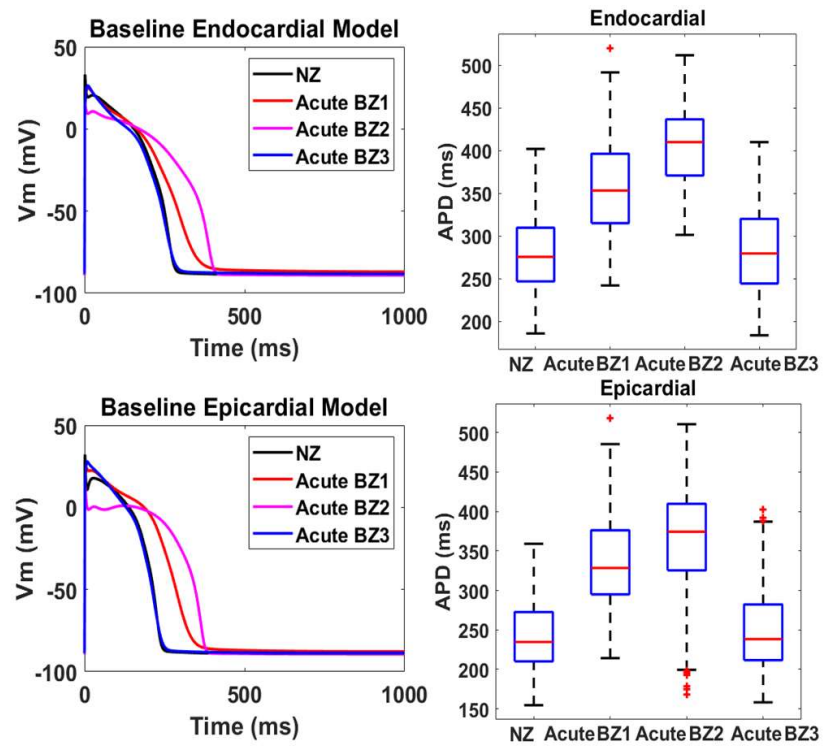

Figure 1. Effects of the three types of acute stage post-MI BZ remodelling on AP. Left: the baseline models; Right: the 253 POMs.
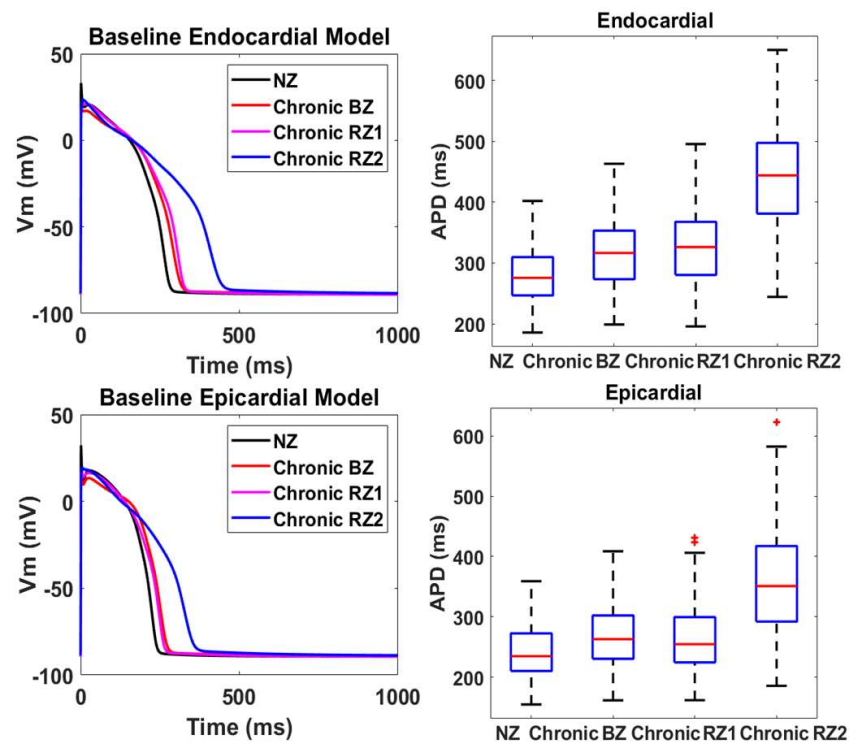

Figure 2. Effects of the chronic stage post-MI BZ and RZ remodelling on AP. Left: the baseline models; Right: the 253 POMs.

\subsection{BZ had weaker calcium transient at both the acute and the chronic post-MI stages}

All three types of acute stage BZ remodelling inhibited calcium transient (CaT) magnitude (CaTmax), with the effect strongest in the Acute BZ2 (Figure 3). The three types of chronic post-MI remodelling also caused weaker CaTmax than NZ, and the CaT was weaker in the Chronic
BZ than the two Chronic RZs (Figure 4). Therefore, at both the acute and chronic post-MI stages, BZ always tended to have the weaker CaT than the corresponding NZ and RZ.
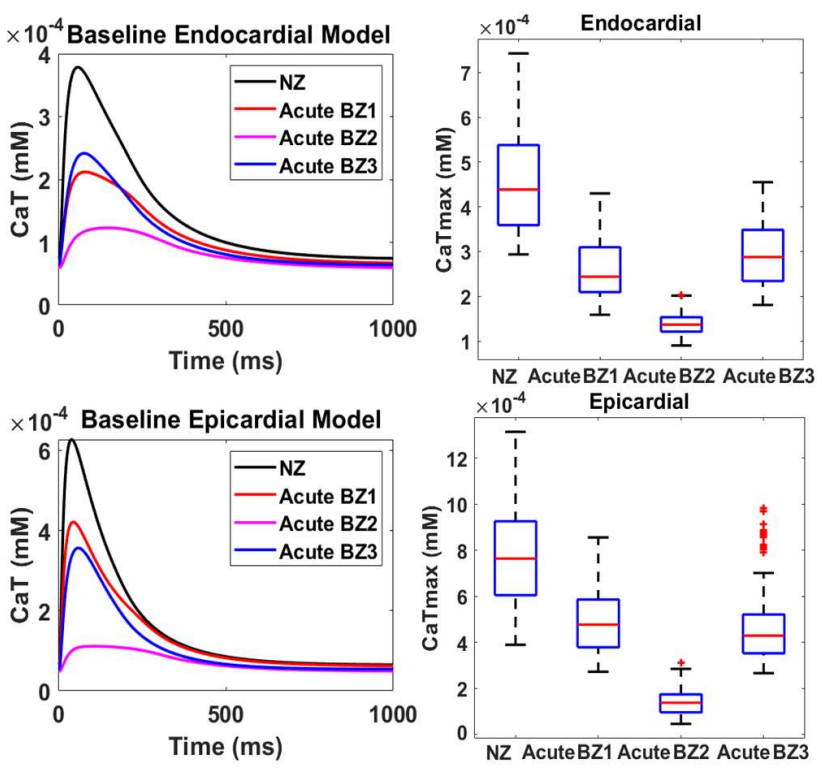

Figure 3. Effects of the three types of acute stage post-MI BZ remodelling on CaT. Left: the baseline models; Right: the 253 POMs.
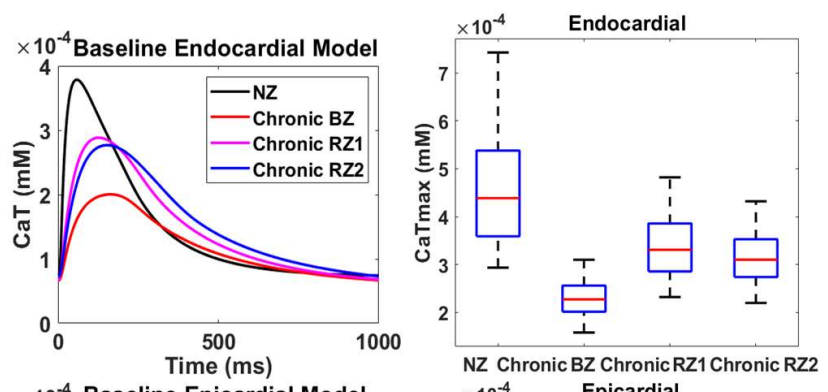

$\times 10^{-4}$ Baseline Epicardial Model
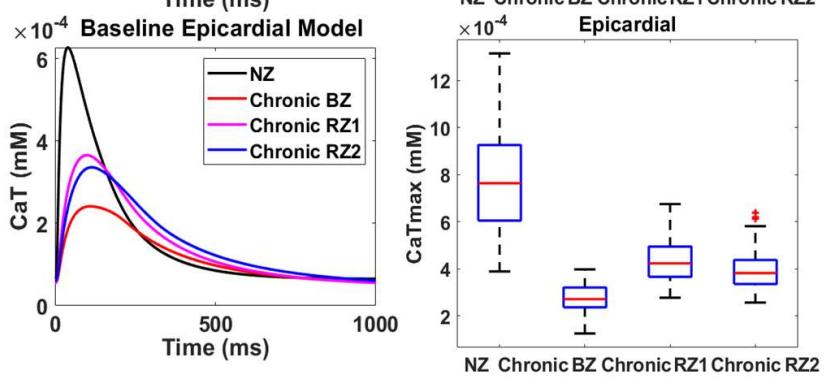

Figure 4. Effects of the chronic stage post-MI BZ and RZ remodelling on CaT. Left: the baseline models; Right: the 253 POMs.

\section{Conclusions}


In this study, we showed for both the acute and chronic post-MI stages, large and small APD prolongations can be present depending on the ionic remodelling implemented. Weak CaT was always observed in the BZ for both stages, which could contribute to stretch induced alterations.

\section{Acknowledgments}

The authors were support by a Wellcome Trust Senior Research Fellowship in Basic Biomedical Sciences $(214290 / Z / 18 / Z)$ to B. Rodriguez.

\section{References}

[1] C. S. Elayi, R. J. Charnigo, P. M. Heron, B. K. Lee, and J. E. Olgin, "Primary prevention of sudden cardiac death early post-myocardial infarction: root cause analysis for implantable cardioverter-defibrillator failure and currently available options," Circ Arrhythm Electrophysiol, vol. 10, no. 6, 2017

[2] J. Pu and P. A. Boyden, "Alterations of $\mathrm{Na}+$ currents in myocytes from epicardial border zone of the infarcted heart. A possible ionic mechanism for reduced excitability and postrepolarization refractoriness," Circ. Res., vol. 81, no. 1, pp. 110-119, Jul. 1997.

[3] R. Aggarwal and P. A. Boyden., "Diminished Ca2+ and $\mathrm{Ba} 2+$ currents in myocytes surviving in the epicardial border zone of the 5-day infarcted canine heart," Circulation Research, vol. 77, no. 6, pp. 1180-1191, Dec. 1995.

[4] M. Jiang, C. Cabo, J. Yao, P. A. Boyden, and G. Tseng, "Delayed rectifier $\mathrm{K}$ currents have reduced amplitudes and altered kinetics in myocytes from infarcted canine ventricle," Cardiovasc. Res., vol. 48, no. 1, pp. 34-43, Oct. 2000.

[5] T. J. Hund et al., "Role of activated CaMKII in abnormal calcium homeostasis and $\mathrm{I}(\mathrm{Na})$ remodeling after myocardial infarction: insights from mathematical modeling," J. Mol. Cell. Cardiol., vol. 45, no. 3, pp. 420428, Sep. 2008.

[6] P. C. Ursell, P. I. Gardner, A. Albala, J. J. Fenoglio, and A. L. Wit, "Structural and electrophysiological changes in the epicardial border zone of canine myocardial infarcts during infarct healing," Circ. Res., vol. 56, no. 3, pp. 436451, Mar. 1985.

[7] B. Hegyi et al., "Complex electrophysiological remodeling in postinfarction ischemic heart failure," Proc. Natl. Acad. Sci. U.S.A., vol. 115, no. 13, pp. E3036-E3044, 272018.

[8] J. Tomek et al., "Development, calibration, and validation of a novel human ventricular myocyte model in health, disease, and drug block," eLife, vol. 8, p. e48890, Dec. 2019.

[9] P. Chang et al., "Heterogeneous upregulation of apaminsensitive potassium currents in failing human ventricles," $J$ Am Heart Assoc, vol. 2, no. 1, Feb. 2013.

[10] X. Zhou et al., "In vivo and in silico investigation into mechanisms of frequency dependence of repolarization alternans in human ventricular cardiomyocytes," Circ.
Res., vol. 118, no. 2, pp. 266-278, Jan. 2016.

[11] O. J. Britton, A. Bueno-Orovio, L. Virág, A. Varró, and B. Rodriguez, "The electrogenic $\mathrm{Na}(+) / \mathrm{K}(+)$ pump is a key determinant of repolarization abnormality susceptibility in human ventricular cardiomyocytes: a population-based simulation study," Front Physiol, vol. 8, p. 278, 2017.

[12] R. Coppini et al., "Late sodium current inhibition reverses electromechanical dysfunction in human hypertrophic cardiomyopathy," Circulation, vol. 127, no. 5, pp. 575584, Feb. 2013.

[13] T. J. Hund et al., "Role of activated CaMKII in abnormal calcium homeostasis and $\mathrm{I}(\mathrm{Na})$ remodeling after myocardial infarction: insights from mathematical modeling," J. Mol. Cell. Cardiol., vol. 45, no. 3, pp. 420 428, Sep. 2008

[14] K. F. Decker and Y. Rudy, "Ionic mechanisms of electrophysiological heterogeneity and conduction block in the infarct border zone," Am. J. Physiol. Heart Circ. Physiol., vol. 299, no. 5, pp. H1588-1597, Nov. 2010,

[15] H. J. Arevalo et al., "Arrhythmia risk stratification of patients after myocardial infarction using personalized heart models," Nat Commun, vol. 7, May 2016.

[16] J. Tomek, B. Rodriguez, G. Bub, and J. Heijman, " $\beta$ adrenergic receptor stimulation inhibits proarrhythmic alternans in postinfarction border zone cardiomyocytes: a computational analysis," Am. J. Physiol. Heart Circ. Physiol., vol. 313, no. 2, pp. H338-H353, Aug. 2017.

[17] C. R. Valdivia et al., "Increased late sodium current in myocytes from a canine heart failure model and from failing human heart," Journal of Molecular and Cellular Cardiology, vol. 38, no. 3, pp. 475-483, Mar. 2005.

[18] M. T. Jiang, A. J. Lokuta, E. F. Farrell, M. R. Wolff, R. A. Haworth, and H. H. Valdivia, "Abnormal $\mathrm{Ca} 2+$ release, but normal ryanodine receptors, in canine and human heart failure," Circ. Res., vol. 91, no. 11, pp. 1015-1022, Nov. 2002.

[19] V. A. Maltsev, N. Silverman, H. N. Sabbah, and A. I. Undrovinas, "Chronic heart failure slows late sodium current in human and canine ventricular myocytes: implications for repolarization variability," European journal of heart failure, vol. 9, no. 3, p. 219, Mar. 2007.

[20] G.-R. Li, C.-P. Lau, T.-K. Leung, and S. Nattel, "Ionic current abnormalities associated with prolonged action potentials in cardiomyocytes from diseased human right ventricles," Heart Rhythm, vol. 1, no. 4, pp. 460-468, Oct. 2004.

[21] R. H. Schwinger et al., "Reduced sodium pump alpha1, alpha3, and beta1-isoform protein levels and $\mathrm{Na}+, \mathrm{K}+-$ ATPase activity but unchanged $\mathrm{Na}+-\mathrm{Ca} 2+$ exchanger protein levels in human heart failure," Circulation, vol. 99, no. 16, pp. 2105-2112, Apr. 1999.

[22] M. A. Høydal et al., "Human cardiomyocyte calcium handling and transverse tubules in mid-stage of postmyocardial-infarction heart failure," ESC Heart Fail, vol. 5, no. 3, pp. 332-342, 2018.

Address for correspondence:

Xin Zhou.

Department of Computer Science, University of Oxford Wolfson Building, Parks Road, Oxford OX1 3QD, UK. xin.zhou@cs.ox.ac.uk 